\title{
A NOTE ON THE LATTICE OF GEOMETRIES
}

\section{JURIS HARTMANIS}

It has been shown that every finite lattice is isomorphic to a sublattice of the lattice of all geometries on some finite set [1]. Since the corresponding problem for partition lattices is still an important unsolved problem we shall investigate in this note the properties of the lattice of all geometries which it shares with the partition lattices [2].

We shall assume familiarity with the definitions and notations of paper [1].

ThEOREM 1. The lattice of all geometries on a set $S$ is complemented.

Proof. Let $G$ be a geometry on $S, G \neq 0$. Let $R$ be a subset of $S$ which has at most two points in common with any line of $G$. Let $\mathfrak{h}$ denote the collection of all such sets and let it be ordered by set inclusion. By the Maximal Principal every chain $C$ of $\mathfrak{h}$ is contained in a maximal chain $\mathfrak{C}$ of $\mathfrak{h}$. It can be seen that $\vee \mathfrak{C}$ is an element of $\mathfrak{h}$. It clearly is a maximal element and let us denote it by $M$. Let the geometry with the only nontrivial line $M$ be denoted by $H$. Then $H \cap G=0$. Let us show that $H \cup G=I$. Let the line of $H \cup G$ which contains $M$ be $L$. Then $H \cup G$ consists of $L$ and the lines of $G$ which are not contained in $L$. We shall show that $L=S$. Let us assume that a point $x$ of $S$ is not in $L$. Since $M$ is a maximal element and $x$ not in $M$ there must exist three distinct points $x, y, z$ which are contained in $M \vee\{x\}$ and some line $t$ of $G$. But then $x, y$ are in $L$ and $t$ and therefore $t \subseteq L$. Thus $L=S$, which completes the proof.

From the proof of Theorem 1 we can see that we proved the somewhat stronger result.

Corollary 1. Let $G$ be a geometry on $S, G \neq I$. Then there exists a geometry $H$ on $S$ which is a complement of $G$, has only one nontrivial line and this line contains any two prescribed points of $S$.

Let two points $a$ and $b$ of $S$ be fixed. Consider the set of those geometries on $S$ which contain at most one nontrivial line and let this line contain $a$ and $b$. This set forms a sublattice of $L G(S)$ which is isomorphic to a Boolean algebra. Thus we obtain

Corollary 2. $L G(S)$ contains a Boolean algebra as a sublattice and every element of $L G(S)$ has a complement in this sublattice.

Presented to the Society, December 29, 1956; received by the editors September 21, 1956. 
CoROllary 3. If a geometry $G$ on $S$ has a unique complement then $G$ is either the zero or the unit element of $L G(S)$.

Proof. Let $G \neq 0, I$. Then $G$ has a line which contains at least three distinct points $a, b, c$. By Corollary 1 there exist two complements of $G$ with only one nontrivial line and these lines contain $a, b$ and $a, c$ respectively. Clearly these two complements are distinct, which proves Corollary 3.

We shall now consider the homomorphisms of $L G(S)$.

LEMMA 1. If $\theta$ is a nontrivial homomorphism on a point lattice $L$, then there exists a point $p$ of $L$ such that $\theta(p)=\theta(0)$.

Proof. Let $\theta$ be a nontrivial homomorphism on $L$. Then there exist two elements $a, b$ in $L, a>b$, such that $\theta(a)=\theta(b)$. Since $L$ is a point lattice there exists a point $p$ such that $a \cap p=p, b \cap p=0$. But then

$$
\theta(p)=\theta(p \cap a)=\theta(p) \cap \theta(a)=\theta(p) \cap \theta(b)=\theta(p \cap b)=\theta(0) .
$$

THEOREM 2. There are only trivial homomorphisms of $L G(S)$.

Proof. Let us consider the geometries on $S$ whose nontrivial lines contain a fixed point $d$ of $S$. The set of all such geometries forms a sublattice $L$ of $L G(S)$. Any two of these nontrivial lines have only the point $d$ in common. Thus after the removal of $d$ the nontrivial lines can be considered as the nontrivial blocks of a partition on the set $S-d$. This yields a one-to-one order preserving mapping of $L$ onto the set of all partitions on $S-d$. Thus $L$ is isomorphic to a partition lattice. Let $\theta$ be a homomorphism which identifies at least two distinct elements of $L G(S)$. Then by Lemma 1 there exists a point $P=\{(a, b, c)\}$ of $L G(S)$ such that $\theta(P)=\theta(0)$. If we set $a=d$ then $P$ is an element of $L$ and thus $\theta$ induces a homomorphism of $L$ which identifies two distinct elements. But Ore [2] proved that there are only trivial homomorphisms on a partition lattice. Thus $\theta$ identifies all elements of $L$. Since $L$ and $L G(S)$ have the same unit and zero elements $\theta$ identifies all elements of $L G(S)$.

THEOREM 3. The group of automorphisms of $L G(S)$ is isomorphic to the symmetric group on $S$.

Proof. Let us denote the geometry with the only nontrivial line $t$ by $\{t\}$. It can be seen that any automorphism of $L G(S)$ maps an element of the form $\{S-p\}, p \in S$, onto some other element of the same form, say $\{S-q\}$. On the other hand any element of $L G(S)$ can be written as a union of elements of the form $\{t\}$. And every such element can be written as an intersection of elements of the form 
$\{S-p\}$. Thus we see that every automorphism is characterized by a permutation of the set of elements of the form $\{S-p\}$ and that any such permutation defines an automorphism. Thus the group of automorphisms is isomorphic to the group of permutations on $S$.

Finally we shall note a difference between the partition lattice and the lattice of all geometries. It can be seen that a quotient lattice of $a$ partition lattice is either isomorphic to a partition lattice or to a direct product of partition lattices. This is important for the embedding problem since it shows that every lattice which can be embedded in a partition lattice can be embedded so that the zero elements coincide. The corresponding result about quotient lattices does not hold for the lattice of all geometries. And in general for the embedding given in [1] the zero elements do not coincide.

\section{REFERENCES}

1. Juris Hartmanis, Two embedding theorems for finite lattices, Proc. Amer. Math. Soc. vol. 7 (1956) pp. 571-577.

2. Oystein Ore, Theory of equivalence relations, Duke Math. J. vol. 9 (1942) pp. 573-627.

Cornell University 